\title{
Randomised Trial of Adjuvant Radiotherapy Following Radical Prostatectomy Versus Radical Prostatectomy Alone in Prostate Cancer Patients with Positive Margins or Extracapsular Extension
}

\author{
Greetta Hackman ${ }^{a}$, Kimmo Taari $^{b}$, Teuvo L. Tammela ${ }^{c}$, Mika Matikainen $^{b}$, Mauri Kouri $^{d}$, \\ Timo Joensuu $^{d, e}$, Tiina Luukkaala ${ }^{f}$, Arto Salonen ${ }^{g}$, Taina Isotalo ${ }^{h}$, Anssi Pétas $^{b}$, Niilo Hendolin $^{i}$, \\ Peter J. Boström ${ }^{j}$, Sirpa Aaltomaa ${ }^{g}$, Kari Lehtoranta ${ }^{h}$, Pekka Hellström ${ }^{k}$, Jarno Riikonen ${ }^{c}$, \\ Merja Korpela ${ }^{l}$, Heikki Minn ${ }^{m}$, Pirkko-Liisa Kellokumpu-Lehtinen ${ }^{n}$, Eero Pukkala ${ }^{o, p}$, \\ Akseli Hemminki ${ }^{d, e, q, *}$, \\ on behalf of the FinnProstate Group
}

\begin{abstract}
${ }^{a}$ Faculty of Medicine, University of Helsinki, Helsinki, Finland; ${ }^{\mathrm{b}}$ Department of Urology, Helsinki University Hospital, Helsinki, Finland; ${ }^{\mathrm{c}}$ Department of Surgery, Tampere University Hospital, Faculty of Medicine and Life Sciences, Tampere University, Tampere, Finland; ${ }^{\mathrm{d}}$ Comprehensive Cancer Center, Helsinki University Hospital, Helsinki, Finland; ${ }^{\mathrm{e}}$ Docrates Cancer Center, Helsinki, Finland; ${ }^{\mathrm{f}}$ Research, Development and Innovation Center, Tampere University Hospital and Health Sciences, Faculty of Social Sciences, Tampere University, Tampere, Finland; ${ }^{\mathrm{g}}$ Department of Urology, Kuopio University Hospital, Kuopio, Finland; ${ }^{\mathrm{h}}$ Department of Urology, Päijät-Häme Central Hospital, Lahti, Finland; ${ }^{\mathrm{i}}$ Department of Surgery, Mikkeli Central Hospital, Mikkeli, Finland; ${ }^{\mathrm{j}}$ Department of Urology, Turku University Hospital, Turku, Finland; ${ }^{\mathrm{k}}$ Department of Urology, Oulu University Hospital, Oulu, Finland; ${ }^{1}$ Department of Oncology and Radiotherapy, Oulu University Hospital, Oulu, Finland; ${ }^{\mathrm{m}}$ Department of Oncology, Turku University Hospital, Turku, Finland; ${ }^{\mathrm{n}}$ Department of Oncology, Tampere University Hospital, Faculty of Medicine and Life Sciences, University of Tampere, Tampere, Finland; ${ }^{\circ}$ Finnish Cancer Registry-Institute for Statistical and Epidemiological Cancer Research, Helsinki, Finland; ${ }^{\mathrm{p}}$ Faculty of Social Sciences, University of Tampere, Tampere, Finland; ${ }^{\mathrm{q}}$ Cancer Gene Therapy Group, Translational Immunology Research Program, University of Helsinki, Helsinki, Finland
\end{abstract}

\section{Article info}

\section{Article history:}

Accepted July 2, 2019

Associate Editor:

T. Morgan

Keywords:

Adjuvant radiotherapy

Extracapsular extension

Prostate cancer

Positive margins

Radical prostatectomy

\begin{abstract}
Background: It remains unclear whether patients with positive surgical margins or extracapsular extension benefit from adjuvant radiotherapy following radical prostatectomy. Objective: To compare the effectiveness and tolerability of adjuvant radiotherapy following radical prostatectomy.

Design, setting, and participants: This was a randomised, open-label, parallel-group trial. A total of 250 patients were enrolled between April 2004 and October 2012 in eight Finnish hospitals, with pT2 with positive margins or pT3a, pN0, M0 cancer without seminal vesicle invasion.

Intervention: A total of 126 patients received adjuvant radiotherapy at $66.6 \mathrm{~Gy}$.

Outcome measurements and statistical analysis: The primary endpoint was biochemical recurrence-free survival, which we analysed using the Kaplan-Meier method and Cox proportional hazard regression. Overall survival, cancer-specific survival, local recurrence, and adverse events were secondary endpoints.

Results and limitations: The median follow-up time for patients who were alive when the follow-up ended was $9.3 \mathrm{yr}$ in the adjuvant group and $8.6 \mathrm{yr}$ in the observation group. The 10-yr survival for biochemical recurrence was $82 \%$ in the adjuvant group and

* Corresponding author. Cancer Gene Therapy Group, University of Helsinki, Haartmaninkatu 3, 00290 Helsinki, Finland. Tel. +358 294125464.

E-mail address: akseli.hemminki@helsinki.fi (A. Hemminki).
\end{abstract}


$61 \%$ in the observation group (hazard ratio [HR] 0.26 [95\% confidence interval $\{\mathrm{CI}\} 0.14-$ 0.48 ], $p<0.001$ ), and for overall survival $92 \%$ and $87 \%$, respectively (HR 0.69 [95\% CI $0.29-1.60], p=0.4$ ). Two and four metastatic cancers occurred, respectively. Out of the 43 patients with biochemical recurrence in the observation group, 37 patients received salvage radiotherapy. In the adjuvant group, 56\% experienced grade 3 adverse events, versus $40 \%$ in the observation group ( $p=0.016$ ). Only one grade 4 adverse event occurred (adjuvant group). A limitation of this study was the number of patients.

Conclusions: Adjuvant radiotherapy following radical prostatectomy is generally well tolerated and prolongs biochemical recurrence-free survival compared with radical prostatectomy alone in patients with positive margins or extracapsular extension.

Patient summary: Radiotherapy given immediately after prostate cancer surgery prolongs prostate-specific antigen progression-free survival, but causes more adverse events, when compared with surgery alone.

(C) 2019 European Association of Urology. Published by Elsevier B.V. All rights reserved.

\section{Introduction}

Curative therapies for clinically localised cT1-2 prostate cancer include radical prostatectomy and radiotherapy, while in locally advanced cT3-4 prostate cancer, there is a lack of general consensus on the risk-benefit ratios of various treatment options [1,2]. The impact of positive surgical margins on survival in localised pT2 after radical prostatectomy is controversial and dependent on other tumour characteristics [3]. Following radical prostatectomy, positive margins or extracapsular extension (pT3-4) are considered independent risk factors for biochemical recurrence [4]. Locally advanced pT3 disease associates with an increased risk of recurrence, metastasis, and prostate cancer death [4,5]. In a multinational study of $>20000$ radical prostatectomy patients, Sooriakumaran et al. [6] reported 14-23\% positive margins and $23-38 \%$ pT3 disease, depending on the surgical technique.

Currently, there is no consensus on the optimal treatment for patients whose disease is pT2 with positive margins or pT3 with/without positive margins, following prostatectomy with curative intent. If these patients benefit from radiotherapy, the question is whether they should receive irradiation in the form of immediate, adjuvant radiotherapy or as salvage radiotherapy given at prostatespecific antigen (PSA) recurrence. The latest guidelines suggest adjuvant radiotherapy or observation following prostatectomy for patients with adverse pathologic findings such as positive surgical margins, seminal vesicle invasion, and/or extracapsular extension [7,8].

Three earlier randomised studies compared adjuvant radiotherapy with observation in radical prostatectomy patients: Southwest Oncology Group (SWOG 8794), European Organization for Research and Treatment of Cancer (EORTC 22911), and German Cancer Society (ARO 96-02/ AUO AP 09/95), all of which included pT3 patients with positive margins [9-11]. In addition, EORTC included pT2 patients with positive margins [10]. Adjuvant radiotherapy compared favourably with observation regarding PSA relapse-free survival in all the studies [9-11]. Only SWOG found benefit in metastasis-free and overall survival [9]. Of note, the majority of events were unrelated to prostate cancer.

While the prior randomised trials have predominantly focused on pT3-4 prostate cancer (with/without positive margins), the design of this study asks whether patients with pT2NOMO (with positive margins) and pT3aNOMO (regardless of margins) benefit from adjuvant radiotherapy following radical prostatectomy.

\section{Patients and methods}

This is a randomised, open-label, parallel-group, multicentre, collaborative study of FinnProstate and Finnish Radiation Oncology Groups (NCT02668718). The Surgical Ethics Committee of Hospital District of Helsinki and Uusimaa evaluated the trial protocol and informed consent form. The trial was performed according to the principles of Declaration of Helsinki. Data are reported on an intent-to-treat basis.

We randomised 250 patients (1:1), with the hypothesis that $80 \%$ in the adjuvant group and $60 \%$ in the observation group will remain biochemical progression free after $2 \mathrm{yr}$ of follow-up, giving a power of $\geq 80 \%$ and significance level of $5 \%$. As calculated by Fischer's exact test, the required sample size for two independent groups was 90 patients/ group. To avoid loss of power due to possible loss in follow-up, investigators writing the protocol decided to increase the sample size to 125 patients/group (39\% safety margin) based on clinical judgement and experience from previous prostate cancer trials.

The inclusion criteria included written informed consent, pT2NOMO with a positive margin or PT3aNOMO (with/without positive margins) prostate cancer, Gleason score 2-10, preoperative PSA $\leq 20 \mu \mathrm{g} / \mathrm{l}$, and postoperative PSA $<0.5 \mu \mathrm{g} / \mathrm{l}$.

The exclusion criteria included other concurrent cancer therapy including systemic endocrine therapy, more than $12 \mathrm{wk}$ since radical prostatectomy, metastatic disease ( $\mathrm{N}+$ or $\mathrm{M} 1)$, and invasion of seminal vesicles.

Following the patient's informed consent, the urologist called Finnish Cancer Registry (Helsinki, Finland), which conducted stratification into three groups by Gleason score (Gleason scores 2-6, 7, and 8-10) and randomisation.

The radiation dose consisted of 66.6 Gy given in 37 fractions of $1.8 \mathrm{~Gy} /$ d, $5 \mathrm{~d}$ per week. Patients received three-dimensional conformal radiation therapy (with linear accelerator $>10 \mathrm{MV}$ ) without pelvic lymph node irradiation.

The protocol defined progression as (1) PSA $>0.4 \mu \mathrm{g} / 1$ in two successive measurements at least $4 \mathrm{wk}$ apart, (2) metastatic prostate cancer, or (3) recurrent prostate cancer in imaging regardless of PSA. In the observation group, salvage radiotherapy could be offered upon disease progression.

We graded adverse events from patients' individual medical records from randomisation to progression or until the last follow-up if the patient was progression free. At visits (between 0 and 51 mo from radical prostatectomy), patients filled three questionnaires: (1) International Index of Erectile Function (IIEF-5), (2) International Prostate Symptom 
Score (IPSS), and (3) Late Effects Normal Tissue Task Force-Subjective, Objective, Management, Analytic (LENT-SOMA) questionnaire with intestinal and urinary questions from the subjective, objective, and management parts of the LENT-SOMA parameters.

We used the Kaplan-Meier method to calculate 10-yr survival rates for biochemical recurrence-free, overall, prostate cancer-specific, metastatic, and castration-resistant prostate cancer (CRPC)-free survival. To test survival difference between the adjuvant and the observation group, we used Cox proportional hazard regression analysis for hazard ratios (HRs) with $95 \%$ confidence intervals (CIs), and for $p$ values. In addition, we used multivariable Cox regression to test (1) the association between biochemical recurrence and group after adjusting for preoperative PSA,
Gleason score (Gleason scores 5-6, 7, and 8-9), and pT stage (pT2, pT3), and (2) the interaction between preoperative PSA and the treatment group regarding biochemical recurrence. Patients were censored at the time of defined event or last follow-up. We used SPSS for the aforementioned analyses (IBM SPSS Statistics for Windows, version 25.0; IBM Corp., Armonk, NY, USA).

We used generalised linear mixed model with lmer function (GLMM) to compare groups (adjuvant and observation) regarding the number of patients experiencing adverse events and the total number of adverse events. In both models, we modelled all adverse events regardless of the grade. We used binary response (any adverse event regardless of the grade vs no adverse event) for adverse events experienced by the

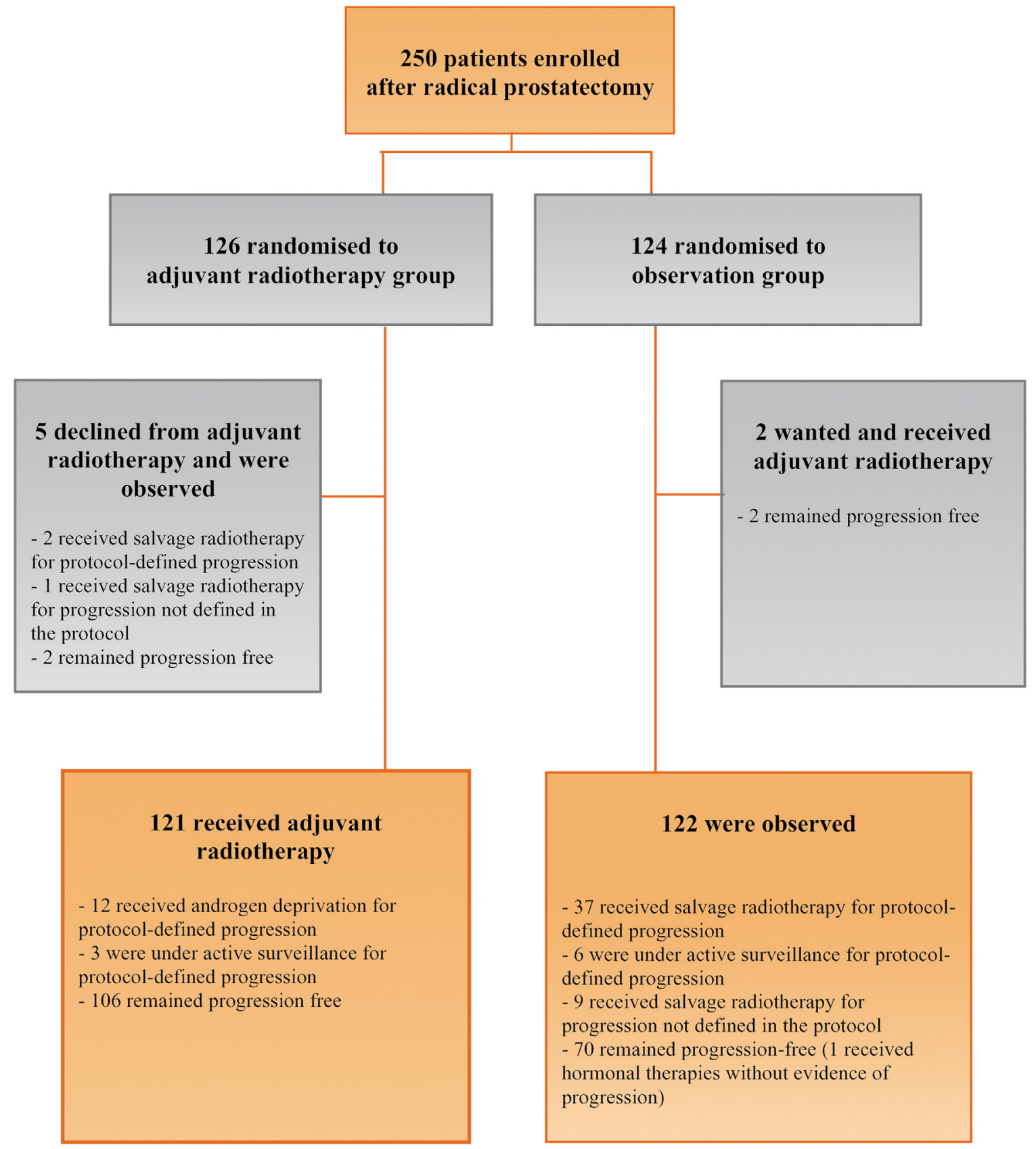

Fig. 1 - Flow chart. Protocol-defined progression = (1) PSA $>0.4 \mu \mathrm{g} / \mathrm{l}$ in two successive measurements at least 4 wk apart, (2) metastatic prostate cancer, or (3) recurrent prostate cancer in imaging regardless of PSA. The median time from radical prostatectomy to initiation of adjuvant radiotherapy was 11.7 wk (range 7.6-30, interquartile range 11-12.6). Five patients had interruptions of adjuvant radiotherapy, two suffered from urinary/intestinal adverse effects, one had viral infection with fever, one had cholecystitis, and for one patient the reason was unavailable. One patient decided to limit the dose to $63 \mathrm{~Gy}$ due to grade 2 faecal frequency. The other four patients received the planned total dose of $66.6 \mathrm{~Gy}$. PSA = prostate-specific antigen. 
patients. The total number of adverse events was modelled as Poisson distribution. In both models, a group (observation vs adjuvant) was modelled as a fixed effect. Patients constituted potential sources of variation, and therefore this subject-specific effect was included as a random effect in the model [12].

In addition, we used a binary response to model severe erectile dysfunction (IIEF-5 scores $1-7$ vs 8-25) and severe urinary symptoms (IPSS scores 20-35 vs 0-19) over follow-up time using GLMM to compare groups (adjuvant and observation). Similarly, we used a binary response to model the severity of LENT-SOMA urinary and intestinal toxicities (grade 3-4 vs 1-2). In these four models, explanatory variables (group and time in months) were modelled as fixed effects, and patient-specific effect as a random effect. Time was treated as a continuous variable. The GLMM was applied with the R statistical software package (version 3.5.2, lme4 function, R Core Team [2018]. R: A language and environment for statistical computing. R Foundation for Statistical Computing, Vienna, Austria. URL https://www.R-project.org/).

Our primary endpoint was biochemical recurrence-free survival. Overall survival, cancer-specific survival, local recurrence, and adverse events were secondary endpoints. Metastatic and castration-resistant survival analyses were unplanned. We measured all survival analyses and follow-up from radical prostatectomy.

\section{Results}

A total of 250 patients enrolled in the trial (Fig. 1) between April 2004 and October 2012 at eight regional Finnish hospitals (listed in the Supplementary material), and the follow-up ended in January 2017. Patient characteristics are given in Table 1. Protocol violations are in listed in Supplementary Table 1.

\subsection{Overall, prostate cancer-specific, and metastatic-free survival}

The median follow-up time for patients who were alive when the follow-up ended was 9.3 yr (range 3.3-12.6, interquartile range [IQR] 6.5-10.3) in the adjuvant group and $8.6 \mathrm{yr}$ (range 3.6-12.1, IQR 6.4-10.4) in the observation group. The $10-\mathrm{yr}$ overall survival was $92 \%$ in the adjuvant group and $87 \%$ in the observation group (HR 0.69 [95\% CI $0.29-1.60$ ], $p=0.4$ ). Only two patients died of prostate cancer, one in each group (Fig. 2 and Table 2). The 10-yr prostate cancer-specific survival was $99 \%$ in both groups (HR 1.00 [95\% CI 0.06-15.91], $p=1$ ).

The 10-yr metastatic-free survival was $98 \%$ in the adjuvant group and $96 \%$ in the observation group (HR 0.49 [95\% CI 0.09-2.68], $p=0.4$ ). The 10-yr CRPC-free survival was $96 \%$ and $92 \%$, respectively (HR 0.50 [95\% CI 0.12-1.88], $p=0.3$ ).

\subsection{Biochemical recurrence and second-line treatments}

The 10 -yr biochemical recurrence-free survival was $82 \%$ in the adjuvant group and $61 \%$ in the observation group (HR 0.26 [95\% CI 0.14-0.48], $p<0.001$ ). When adjusted for preoperative PSA, Gleason score, and pT stage, the HR for biochemical recurrence for adjuvant versus observation group was 0.30 (95\% CI 0.16-0.54, $p<0.001$ ). The number needed to treat was 4 . The median follow-up time for biochemical recurrence-free patients was $8.6 \mathrm{yr}$ in the
Table 1 - Patient characteristics (number of patients) at randomisation in the adjuvant radiotherapy and observation groups

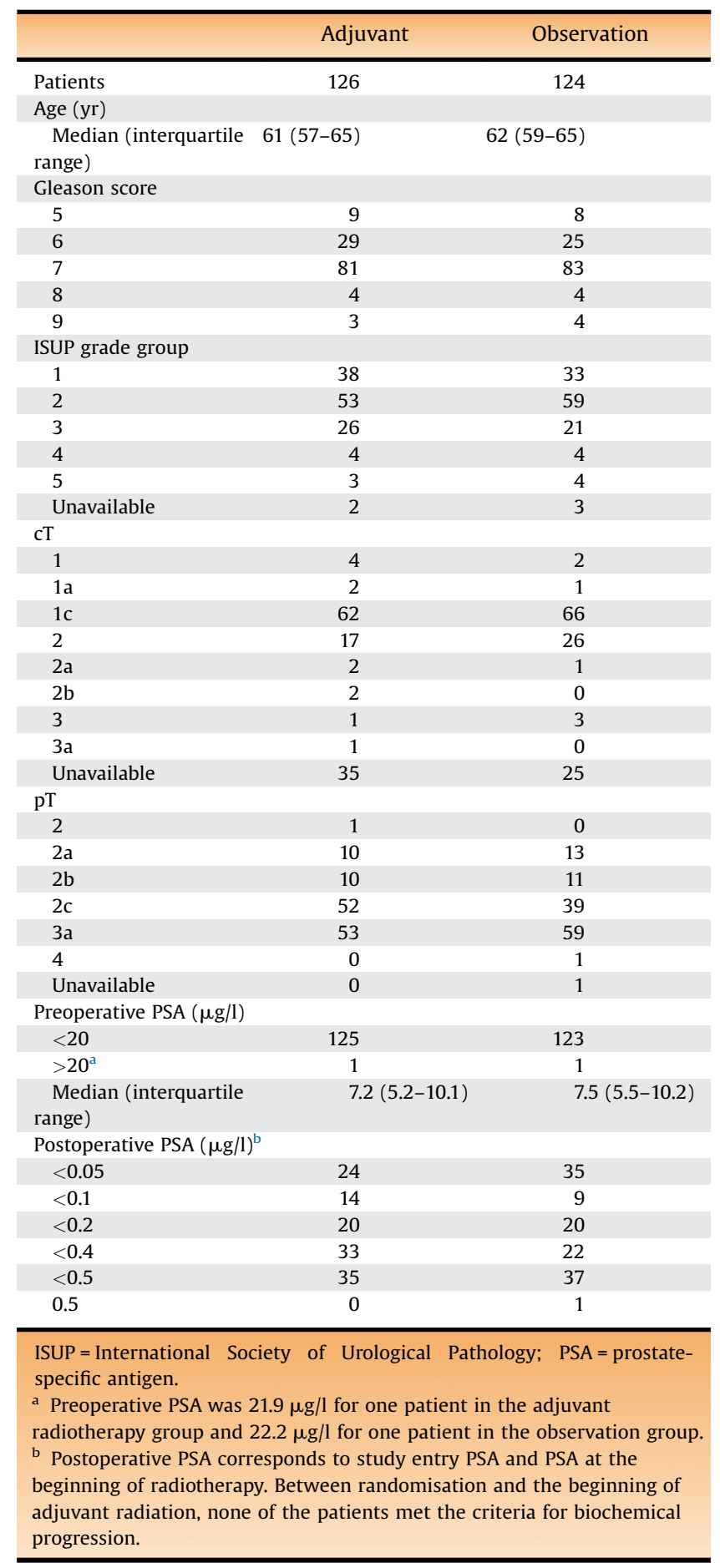

adjuvant group and $8.2 \mathrm{yr}$ in the observation group. We present the median preoperative PSA for patients with/ without biochemical recurrence in Supplementary Table 2.

In the adjuvant group, three of 73 patients with pT2 disease and positive surgical margins experienced protocoldefined biochemical recurrence compared with 21 of 63 in the observation group. For pT3 patients, the numbers were $12 / 53$ and 22/59, respectively. For Gleason score 5-6 
A

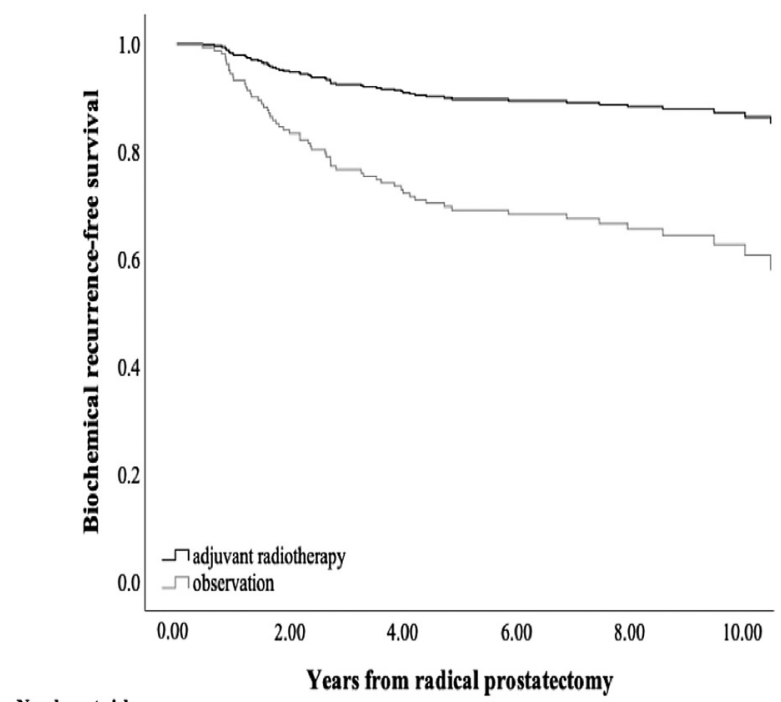

Number at risk

$\begin{array}{lllllll}\text { Adjuvant } & 126 & 121 & 116 & 112 & 112 & 112 \\ \text { Observation } & 124 & 102 & 90 & 85 & 81 & 75\end{array}$

C

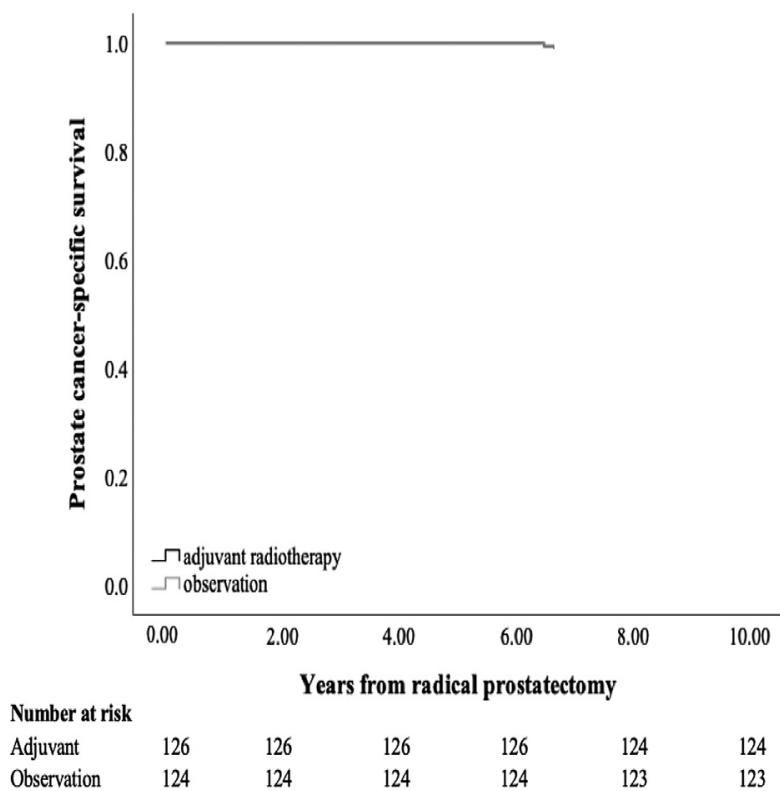

$\begin{array}{lllllll}\text { Observation } & 124 & 124 & 124 & 124 & 123 & 123\end{array}$
B

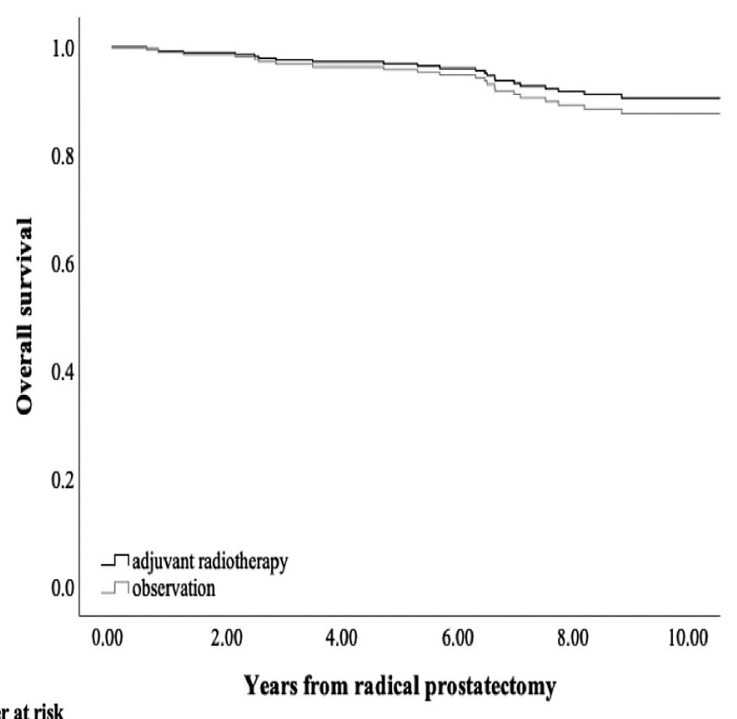

Number at risk

$\begin{array}{lllllll}\text { Adjuvant } & 126 & 124 & 122 & 121 & 115 & 115\end{array}$

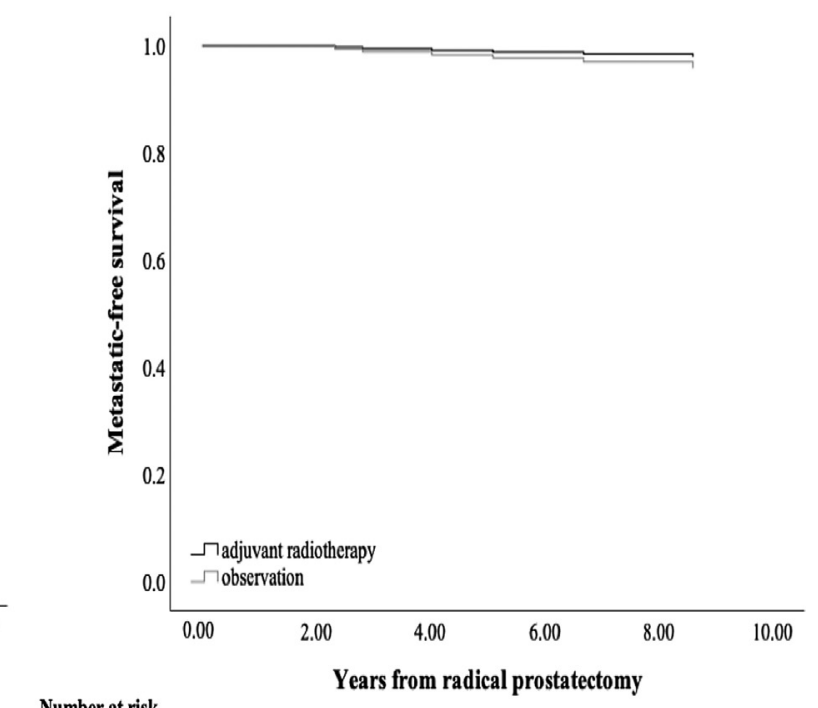

Number at risk

$\begin{array}{lllllll}\text { Adjuvant } & 126 & 126 & 125 & 125 & 125 & 123\end{array}$

Fig. 2 - Crude Cox proportional hazard regression for biochemical recurrence-free, overall, prostate cancer-specific, and metastatic-free survival in the adjuvant radiotherapy and the observation group. (A) Biochemical recurrence-fee survival. (B) Overall survival. (C) Prostate cancer-specific survival. (D) Metastasis-free survival.

patients, two of 38 in the adjuvant group experienced protocol-defined biochemical recurrence compared with 12 of 33 in the observation group. For Gleason score 7, the numbers were 11 of 81 and 29 of 83 patients, respectively.

In the adjuvant group, 12 patients received androgen deprivation as second-line treatment (median PSA $1.4 \mu \mathrm{g} / \mathrm{l}$, range $0.44-32$, IQR $0.78-5.7$ ) and three were under active surveillance after protocol-defined progression (Fig. 1). Out of the 43 patients with protocol-defined biochemical recurrence in the observation group, 37 received salvage radiotherapy within a median of $20 \mathrm{wk}$ (range 4.6-152, IQR 12-33) from progression (median PSA $0.7 \mu \mathrm{g} / \mathrm{l}$, range $0.42-$ 8.2, IQR 0.57-0.83; Supplementary Fig. 1). PSA was measured a median of $4.7 \mathrm{wk}$ (range $0-12$, IQR $1.1-7.5$ ) 
Table 2 - Main results for the adjuvant radiotherapy versus the observation group: number of patients experiencing protocol-defined biochemical recurrence, metastatic prostate cancer, castration-resistant prostate cancer, prostate cancer death, and death from any cause ${ }^{a}$

\begin{tabular}{|c|c|c|c|c|}
\hline & $\begin{array}{l}\text { Adjuvant } \\
(n=126)\end{array}$ & $\begin{array}{l}\text { Observation } \\
\quad(n=124)\end{array}$ & $\mathrm{HR}, 95 \% \mathrm{CI}$ & $p$ value \\
\hline Biochemical recurrence $^{\mathrm{b}}$ & 15 & 43 & $0.30,0.16-0.53$ & $<0.001$ \\
\hline Metastatic & 2 & 4 & $0.49,0.09-2.68$ & 0.4 \\
\hline Castration resistant ${ }^{\mathrm{C}}$ & 3 & 6 & $0.47,0.12-1.88$ & 0.3 \\
\hline Prostate cancer death $^{\mathrm{d}}$ & 1 & 1 & $1.00,0.06-15.91$ & 1 \\
\hline Death from any cause & 10 & 13 & $0.76,0.33-1.72$ & 0.5 \\
\hline \multicolumn{5}{|c|}{$\begin{array}{l}\mathrm{CI}=\text { confidence interval; } \mathrm{HR}=\text { hazard ratio; } \mathrm{PSA}=\text { prostate-specific antigen. } \\
\text { a Separate univariable Cox proportional hazard regression analysis was used showing results by HRs with } 95 \% \text { CIs, and } p \text { values, associated with receiving } \\
\text { adjuvant radiotherapy versus observation. } \\
\text { b We detected no local recurrence with imaging, and all patients diagnosed with metastases experienced biochemical progression before metastases were } \\
\text { detected. } \\
\text { c Castration-resistant cancer was defined as consecutively increasing PSA in two successive measurements at least } 4 \text { wk apart despite androgen deprivation } \\
\text { therapy. } \\
\text { d These patients both had Gleason } 7(4+3) \text { prostate cancer; they died } 6.7 \text { and } 6.5 \mathrm{yr} \text { after prostatectomy at the age of } 62 \text { and } 53 \text { yr with metastatic-free survival of } \\
2.8 \text { and } 2.3 \mathrm{yr} \text { (adjuvant radiotherapy and observation group, respectively). For the other four patients with metastases, the range of metastatic-free survival was } \\
4-8.6 \mathrm{yr} \text {. }\end{array}$} \\
\hline
\end{tabular}

before the beginning of radiation. Six patients continued active surveillance (Fig. 1). Following salvage radiotherapy, 28 patients achieved PSA remission, while nine required systemic therapies.

In addition, 10 patients received salvage radiotherapy for biochemical recurrence that did not meet the protocol's criteria for progression, with a median PSA value of $0.4 \mu \mathrm{g} / \mathrm{l}$ (range 0.12-0.53, IQR 0.18-0.43; Fig. 1 and Supplementary Table 1). One was from the adjuvant group, but had declined adjuvant radiotherapy, while nine were from the observation group. Of these 10 patients, all except one (from the observation group) achieved PSA remission. Additionally, one patient in the observation group, with no PSA progression or metastases, received hormonal therapies.

\subsection{Toxicity}

The most common grade 3 adverse events were erectile dysfunction (37\% of adjuvant and $28 \%$ of observation patients) and urinary incontinence (12\% and $5 \%$, respectively; Table 3). One grade 4 adverse event, a compartment syndrome, occurred in the adjuvant group. This was a postoperative complication of cystectomy occurring nearly $7 \mathrm{yr}$ after radical prostatectomy. Prior to cystectomy, the patient suffered from incontinence following transurethral incision of the vesicourethral anastomosis done due to urinary retention, a postoperative complication of ventral hernia repair. There were no grade 5 adverse events.

IIEF-5, IPSS, and LENT-SOMA results are given in Fig. 3.

\section{Discussion}

There was a clear statistically significant difference in biochemical recurrence-free survival, which is in accordance with earlier findings [9-11]. The most notable differences were seen among patients with (1) pT2 disease and positive margins, and (2) Gleason scores 5-6.
A lack of conclusive results from randomised trials comparing adjuvant radiotherapy and observation, fear of toxicity, and avoidance of "overtreatment" have promoted active surveillance and early salvage irradiation, resulting in low use of adjuvant radiotherapy even in patients with adverse pathologic features [13]. Results from retrospective studies comparing adjuvant radiotherapy and early salvage radiation are controversial, while results from several ongoing randomised trials (RADICALS and RAVES) are not available yet [14-16].

When comparing early and late salvage radiotherapy following radical prostatectomy, the observational data support improved efficacy with early irradiation [17]. The common definition for early and late salvage radiation is PSA $\leq 0.5 \mu \mathrm{g} / \mathrm{l}$ and PSA $>0.5 \mu \mathrm{g} / \mathrm{l}$, respectively [17]. Since in this trial salvage radiotherapy in the observation group was given at a median PSA of $0.7 \mu \mathrm{g} / \mathrm{l}$, this would be considered as late salvage, although it was lower compared with SWOG, EORTC, and ARO, which had the median PSA levels of 0.75-1, 1.7, and $1.7 \mu \mathrm{g} / \mathrm{l}$, respectively [9-11].

According to contemporary standards, the enrolment criteria for postoperative PSA would have been $<0.2 \mu \mathrm{g} / \mathrm{l}$, compared with our threshold of PSA $<0.5 \mu \mathrm{g} / \mathrm{l}$, which was standard when the trial was designed. However, $46 \%$ of patients in the adjuvant group and $52 \%$ in the observation group had preoperative PSA $<0.2 \mu \mathrm{g} / \mathrm{l}$.

In addition, at the initiation of this trial, a common threshold for biochemical recurrence was PSA $>0.4 \mu \mathrm{g} / \mathrm{l}$, which was therefore applied in this protocol. However, due to more accurate testing, currently PSA $\geq 0.2 \mu \mathrm{g} / 1$ is frequently used, which explains why some patients in this trial received second-line therapy with PSA levels $<0.4 \mu \mathrm{g} / \mathrm{l}$.

Compared with this study, the other three randomised trials included patients with slightly more adverse features. In SWOG, 33\% had seminal vesicle invasion alone or together with positive margins and extracapsular extension, and $15 \%$ had postoperative PSA $\geq 0.2 \mu \mathrm{g} / \mathrm{l}$ [9]. In EORTC, $25 \%$ had seminal vesicle invasion, and $30 \%$ 
Table 3 - Number (and proportion) of patients in the adjuvant radiotherapy and the observation group experiencing adverse events, scored from patients' individual medical records, and the three most common disorders ${ }^{\mathrm{a}, \mathrm{b}, \mathrm{c}}$

\begin{tabular}{|c|c|c|c|c|c|}
\hline & \multirow{2}{*}{$\begin{array}{l}\text { Adjuvant }(N=126) \\
n(\%)\end{array}$} & \multirow{2}{*}{$\begin{array}{l}\text { Observation }(N=124) \\
n(\%)\end{array}$} & \multicolumn{3}{|c|}{$\begin{array}{l}\text { Univariable observation vs } \\
\text { adjuvant (any adverse event } \\
\text { regardless of the grade) }\end{array}$} \\
\hline & & & $p$ value & OR & $95 \% \mathrm{CI}$ \\
\hline Number of patients experiencing adverse event & & & $0.009^{\mathrm{e}}$ & 0.71 & $(0.55-0.92)$ \\
\hline Grade 1 & $121(96)$ & $105(85)$ & & & \\
\hline Grade 2 & $115(91)$ & $107(87)$ & & & \\
\hline Grade 3 & $70(56)$ & $50(40)$ & & & \\
\hline Grade 4 & $1(1)$ & $0(0)$ & & & \\
\hline Number of patients experiencing gastrointestinal disorders & & & $<0.001^{\mathrm{e}}$ & 0.12 & $(0.07-0.19)$ \\
\hline Grade 1 & $97(77)$ & $16(13)$ & & & \\
\hline Grade 2 & $29(23)$ & $4(3)$ & & & \\
\hline Grade 3 & $1(1)$ & $1(1)$ & & & \\
\hline Grade 4 & $0(0)$ & $0(0)$ & & & \\
\hline Number of patients experiencing urinary disorders & & & $<0.001^{\mathrm{e}}$ & 0.48 & $(0.36-0.64)$ \\
\hline Grade 1 & $111(88)$ & $77(62)$ & & & \\
\hline Grade 2 & $72(57)$ & $47(38)$ & & & \\
\hline Grade 3 & $18(14)$ & $7(6)$ & & & \\
\hline Grade 4 & $0(0)$ & $0(0)$ & & & \\
\hline Number of patients experiencing erectile dysfunction & & & $0.050^{\mathrm{e}}$ & 0.75 & $(0.56-1.00)$ \\
\hline Grade 1 & $71(56)$ & $52(42)$ & & & \\
\hline Grade 2 & $94(75)$ & $95(77)$ & & & \\
\hline Grade 3 & $47(37)$ & $35(28)$ & & & \\
\hline Grade 4 & $0(0)$ & $0(0)$ & & & \\
\hline Total number of adverse events & & & $<0.001^{\mathrm{f}}$ & & \\
\hline Grade 1 & 733 & 259 & & & \\
\hline Grade 2 & 298 & 165 & & & \\
\hline Grade 3 & 105 & 62 & & & \\
\hline Grade 4 & 1 & 0 & & & \\
\hline Total & 1137 & 486 & & & \\
\hline \multicolumn{6}{|l|}{ Median and range of adverse events per patient } \\
\hline Grade 1 & $6(0-17)$ & $1.5(0-11)$ & & & \\
\hline Grade 2 & $2(0-14)$ & $1(0-4)$ & & & \\
\hline Grade 3 & $1(0-6)$ & $0(0-3)$ & & & \\
\hline Grade 4 & $0(0-1)$ & - & & & \\
\hline \multicolumn{6}{|c|}{$\begin{array}{l}\mathrm{CI}=\text { confidence interval; OR = odds ratio PSA = prostate-specific antigen. } \\
\text { a Adverse events were scored from individual medical records using Common Toxicity Criteria for Adverse Events version } 4.03 \text {. The scoring of adverse events } \\
\text { began from randomisation and ended when progression occurred or at the end of the follow-up time if the patient was PSA progression free. We included all } \\
\text { adverse events; that is, the relation between the adverse event and the trial treatment was not evaluated. } \\
\text { b Two patients (one from each group) experienced no adverse event. } \\
\text { c Patients with grade } 3 \text { urinary incontinence required surgical treatment including transurethral injection, urethrotomy, sling, and artificial urinary sphincter. } \\
\text { Twelve patients in the adjuvant group and three in the observation group suffered grade } 3 \text { urethral stricture, which needed surgical intervention. For grade } \\
3 \text { inguinal hernia requiring surgical intervention, the numbers were } 8 \text { and 9, respectively. } \\
\text { d Univariable generalised linear mixed model: } \\
\text { e binomial (any adverse event regardless of the grade vs no grade) and } \\
\text { f Poisson distribution. In both models, group (observation vs adjuvant) was modelled as a fixed effect and patients as a random effect. }\end{array}$} \\
\hline
\end{tabular}

had postoperative PSA $\geq 0.2 \mu \mathrm{g} / \mathrm{l}[10]$. In ARO, 49\% had $>$ pT3a cancer [11]. The aforementioned adverse features are often considered an indication for adjuvant radiotherapy. For example, $80-86 \%$ of patients with seminal vesicle invasion suffer from PSA recurrence $[18,19]$. Hence, we excluded these patients.

In a subgroup analysis of EORTC, positive surgical margins and age $<70 \mathrm{yr}$ were associated with greater benefit from adjuvant radiotherapy [10]. In ARO, patients with positive surgical margins benefitted most from adjuvant radiotherapy [11]. SWOG reported no subgroups that benefitted significantly from adjuvant radiotherapy [9].

There were significantly more adverse events in the adjuvant group, as expected, although most of these were grade 1-2 and transient. The findings were similar regarding patient-reported toxicities (LENT-SOMA), although the patients reported more grade 3-4 LENT-SOMA toxicities than Common Terminology Criteria for Adverse Events (CTCAE, version 4.03). When comparing the toxicity grading used in this study, LENT-SOMA had a tendency for higher grades compared with CTCAE. Depending on the time of visit, a median of 86 (range 18-105) patients in the adjuvant group and a median of 71 (range 28-94) patients in the observation group filled the LENT-SOMA questionnaire (Fig. 3). The compliance rate of patient-reported outcomes often declines towards the end of the trial $[20,21]$. We detected no trends between treatment groups or hospitals regarding the compliance rate.

Following radical prostatectomy, the $10-\mathrm{yr}$ overall survival varies from $72 \%$ to $87 \%$ in different studies 
A

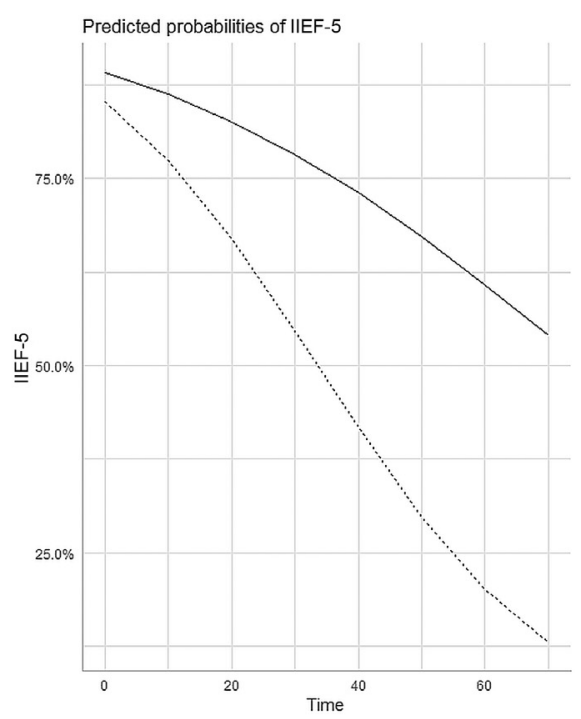

C
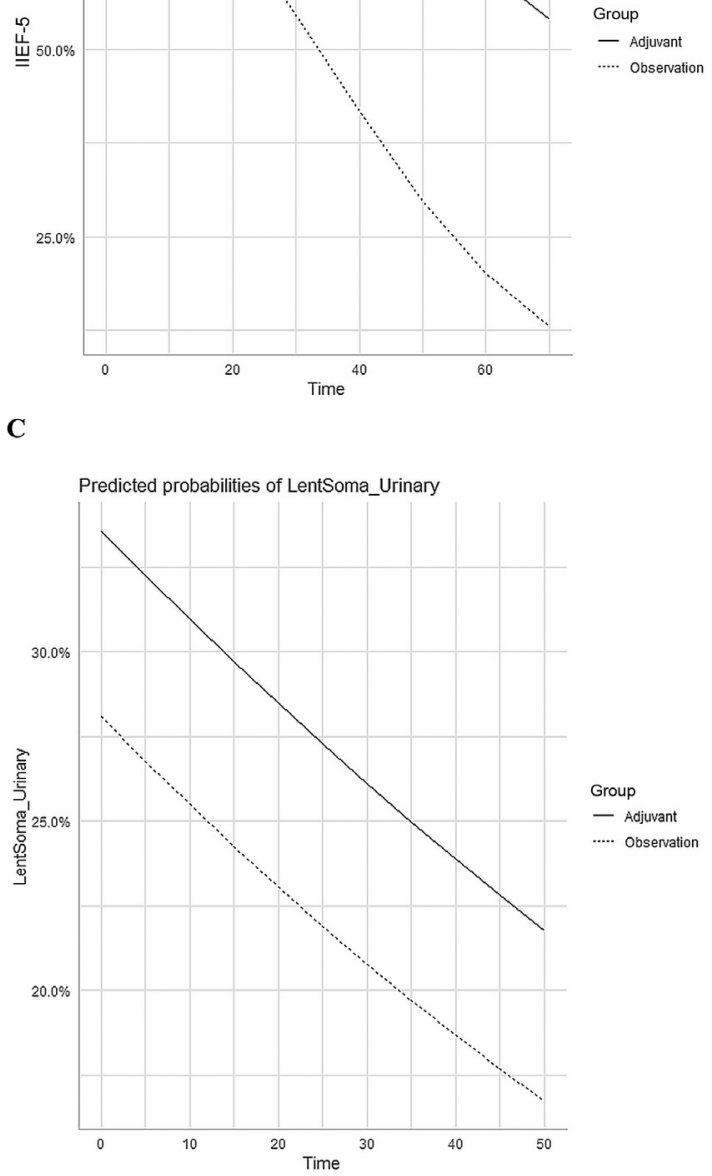

B

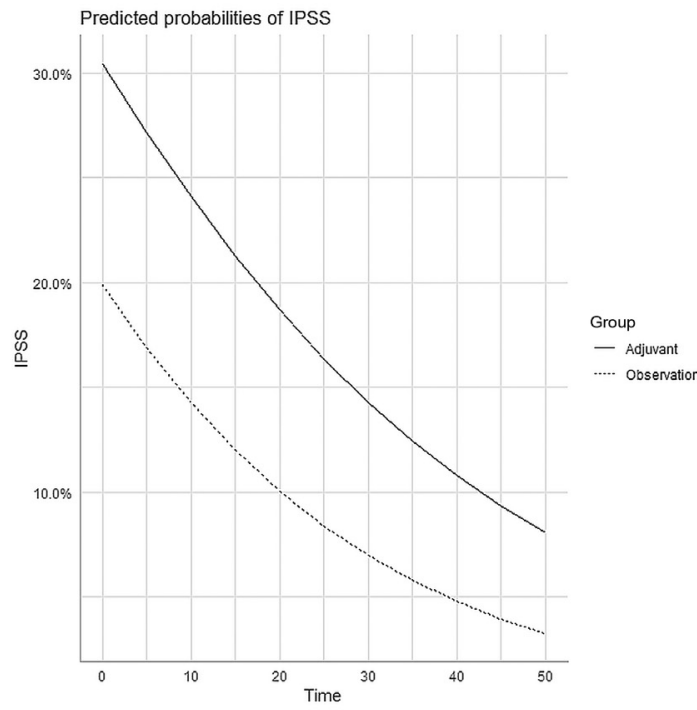

D

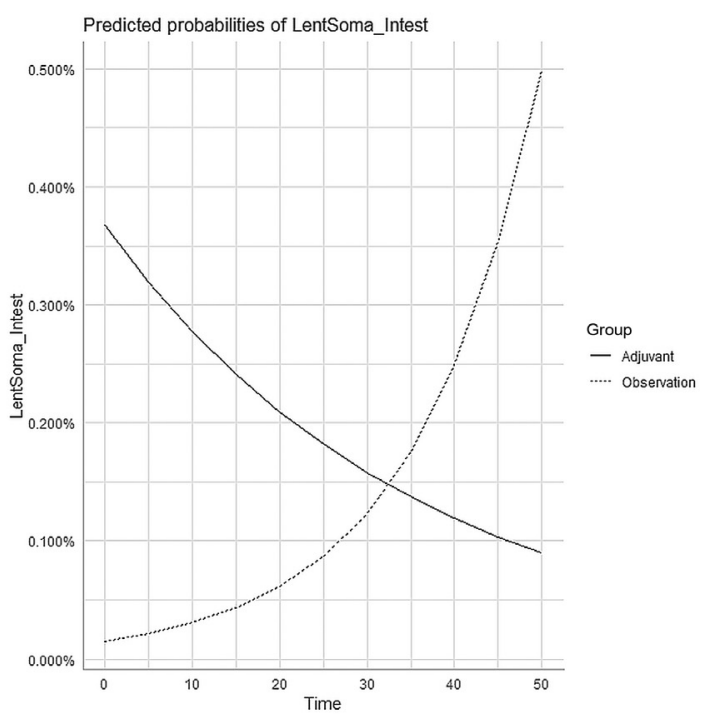

Fig. 3 - (A) International Index of Erectile Function (IIEF-5), (B) International Prostate Symptom Score (IPSS), and maximum Late Effects Normal Tissue Task Force-Subjective, Objective, Management, Analytic (LENT-SOMA) grade for (C) urinary and (D) intestinal toxicities measured from radical prostatectomy and presented separately for the adjuvant radiotherapy and the observation group as predicted probabilities using a generalised linear mixed model (GLMM). (A) Severity of erectile dysfunction. Predicted probabilities of IIEF-5 shown separately for the adjuvant and the observation group. Effect of treatment group (observation vs adjuvant; OR 0.70 [95\% $\mathrm{Cl} 0.29-1.68], p=0.4)$ and time in months $(\mathrm{OR}$ 0.97 [95\% CI 0.95-0.99], $p=0.002)$, and interaction of group and time (OR 0.98 [95\% CI $0.95-1.00], p=0.047)$ for severe dysfunction (IIEF-5 score was modelled as binomially distributed scores 1-7 vs 8-25) over continuous time (months) according to GLMM. IIEF-5 score: 1-7 = severe erectile dysfunction, 8-21 = mildmoderate erectile dysfunction, and 22-25 = no erectile dysfunction. (B) Severity of urinary symptoms. Predicted probabilities of IPSS shown separately for the adjuvant and the observation group. Effect of treatment group (observation vs adjuvant; OR 0.51 [95\% $\mathrm{CI} 0.25-1.03], p=0.061$ ) and time in months (OR 0.96 [95\% CI 0.95-0.98], $p<0.001$ ) for severe dysfunction (IPSS score was modelled as binomially distributed scores 20 -35 vs 0-19) over continuous time (months) according to GLMM. IPSS score: $0-7=$ mild urinary symptoms, 8-19 $=$ moderate urinary symptoms, and $20-35=$ severe urinary symptoms. (C) Maximum LENT-SOMA grade for each patient, urinary toxicities. Predicted probabilities of LENT-SOMA grades shown separately for the adjuvant and the observation group. Effect of treatment group (observation vs adjuvant; OR 0.76 [95\% CI 0.40-1.42], $p=0.4$ ) and time in months (OR 0.99 [95\% CI 0.97-1.00], $p=0.064$ ) for severe grades (urinary toxicities were modelled as binomially distributed grades 3-4 vs 0-2) over continuous time (months) according to GLMM. (D) Maximum LENT-SOMA grade for each patient, intestinal toxicities. Predicted probabilities of LENTSOMA grades shown separately for the adjuvant and the observation group. Effect of treatment group (observation vs adjuvant; OR 0.04 [95\% CI 0.000.43 ], $p=0.008$ ), time in months (OR 0.97 [95\% CI 0.94-1.01], $p=0.1$ ), and interaction (OR 1.10 [95\% CI 1.03-1.18], $p=0.006)$ for severe grades (LENTSOMA modelled as binomially distributed grades 3-4 vs 0-2) over continuous time (months) according to GLMM. The LENT-SOMA toxicities were graded according to the patients' answers from 0 to 4 , where grade 0 stands for no toxicity and grade 4 stands for the most severe toxicity. For one LENT-SOMA question regarding the management of dysuria, the answer option for surgical intervention (grade 4 toxicity) was unavailable; therefore, the answers for this question were graded from 1 to 3 . The most common LENT-SOMA toxicities were urinary frequency (93\% of the patients in the adjuvant group and $92 \%$ in the observation group filled the questionnaire), urinary incontinence $(70 \%$ and $62 \%$, respectively), decreased urinary stream (61\% and 56\%, respectively), and rectal tenesmus (64\% and $42 \%$, respectively). The most common grade 4 toxicities were kidney-related toxicity (18 patients in the adjuvant group and 15 in the observation group), urinary incontinence (seven and five patients, respectively), and urinary frequency (five and two patients, respectively). The most common grade 4 kidney-related toxicity was based on two questions: answering "yes" to "do you suffer from tiredness and headache?" led to grade 3, and "yes" to "are you passing less urine than you usually do/are your feet swollen?" led to grade 4 toxicity. $95 \% \mathrm{CI}=95 \%$ confidence interval; $O \mathrm{OR}=$ odds ratio. 
[22,23]. Metastatic disease and thus prostate cancer death can occur long after biochemical recurrence, suggesting that considerably longer follow-up time would be required to detect any survival benefit.

\section{Conclusions}

In summary, adjuvant radiotherapy prolongs the time from radical prostatectomy to biochemical recurrence with the strongest impact on pT2 disease with positive margins and Gleason score 5-7. However, adjuvant radiotherapy causes more adverse effects compared with observation, and salvage therapy upon biochemical recurrence appears as effective as adjuvant therapy with regard to overall survival. In the observation arm, 37 of 124 patients received salvage radiotherapy for protocol-defined progression, after which 28 remained recurrence free, while 121 of 126 patients in the adjuvant arm received radiotherapy following radical prostatectomy (five declined radiation despite randomisation into this arm). Of note, more cases of metastatic disease and CRPC occurred in the observation ("salvage") arm, suggesting that high-risk patients should be offered the possibility to consider adjuvant radiotherapy following radical prostatectomy. Only the patient can balance the subjective questions of radiation-related adverse events to a lower risk of biochemical recurrence, cancer progression, and its consequences.

Author contributions: Akseli Hemminki had full access to all the data in the study and takes responsibility for the integrity of the data and the accuracy of the data analysis.

Study concept and design: Tammela, Joensuu, Kouri, Salonen, Aaltomaa, Lehtoranta, Hellström, Korpela, Minn, Kellokumpu-Lehtinen, Pukkala.

Acquisition of data: Taari, Tammela, Matikainen, Kouri, Joensuu, Salonen, Isotalo, Pétas, Hendolin, Boström, Aaltomaa, Lehtoranta, Hellström, Riikonen, Korpela, Minn, Kellokumpu-Lehtinen, Pukkala.

Analysis and interpretation of data: Hackman, Taari, Hemminki.

Drafting of the manuscript: Hackman, Taari, Hemminki.

Critical revision of the manuscript for important intellectual content: Hackman, Taari, Tammela, Matikainen, Kouri, Joensuu, Luukkaala, Salonen, Isotalo, Pétas, Hendolin, Boström, Aaltomaa, Lehtoranta, Hellström, Riikonen, Korpela, Minn, Kellokumpu-Lehtinen, Pukkala, Hemminki.

Statistical analysis: Hackman, Luukkaala.

Obtaining funding: Hackman, Hemminki.

Administrative, technical, or material support: None.

Supervision: Taari, Hemminki.

Other: None.

Financial disclosures: Akseli Hemminki certifies that all conflicts of interest, including specific financial interests and relationships and affiliations relevant to the subject matter or materials discussed in the manuscript (eg, employment/affiliation, grants or funding, consultancies, honoraria, stock ownership or options, expert testimony, royalties, or patents filed, received, or pending), are the following: G. Hackman is a shareholder in Astra Zeneca, and has taken part in congress with support from Janssen. K. Taari has received research funding from Medivation/ Astellas/Pfizer, Orion, and Myovant. T.L. Tammela has received consultancy honoraria from Astellas, Bayer, Janssen-Cilag, and Lidds Ab. A. Salonen has taken part in congress with support from Astellas Pharma, Swanmedica, and Sanofi; and received consultant honoraria from
Janssen and Amgen. P. Hellström has taken part in congress with support from Swanmedica. M. Korpela has taken part in congress with support from Merck. P.-L. Kellokumpu-Lehtinen has received funding from Sanofi, Merck, Lilly, Bayer, Pfizer, and Roche. A. Hemminki is a shareholder in Targovax ASA. A. Hemminki is an employee and a shareholder in TILT Biotherapeutics Ltd. and Aeruginosa Oy. A. Hemminki has consulted for Amgen Inc.

Funding/Support and role of the sponsor: This study was supported by The Finnish Medical Foundation, Finnish Cancer Organizations, Ida Montini Foundation, Jane and Aatos Erkko Foundation, HUCH Research Funds (EVO), Sigrid Juselius Foundation, and University of Helsinki.

Acknowledgements: We thank the following research nurses: Merja Rignell (Helsinki University Hospital), Tuula Kivimaa (Oulu University Hospital), Maria Pekki (Tampere University Hospital), Tuula Gavrilov (North Carelia Central Hospital), Jaana Seppänen (Kuopio University Hospital), and Hanna-Kaisa Huhtala (Turku University Hospital).

\section{Appendix A. Supplementary data}

Supplementary data associated with this article can be found, in the online version, at https://doi.org/10.1016/j. eururo.2019.07.001.

\section{References}

[1] Hamdy FC, Donovan JL, Lane JA, et al. 10-Year outcomes after monitoring, surgery, or radiotherapy for localized prostate cancer. N Engl J Med 2016;375:1415-24.

[2] Ahlgren GM, Flodgren P, Tammela TLJ, et al. Docetaxel versus surveillance after radical prostatectomy for high-risk prostate cancer: results from the prospective randomised, open-label phase 3 Scandinavian Prostate Cancer Group 12 trial. Eur Urol 2018;73:870-6.

[3] Chapin BF, Nguyen JN, Achim MF, et al. Positive margin length and highest Gleason grade of tumor at the margin predict for biochemical recurrence after radical prostatectomy in patients with organconfined prostate cancer. Prostate Cancer Prostatic Dis 2018;21:221-7.

[4] Suardi N, Ficarra V, Willemsen P, et al. Long-term biochemical recurrence rates after robot-assisted radical prostatectomy: analysis of a single-center series of patients with a minimum follow-up of 5 years. Urology 2012;79:133-8.

[5] Shipley WU, Seiferheld W, Lukka HR, et al. Radiation with or without antiandrogen therapy in recurrent prostate cancer. $\mathrm{N}$ Engl J Med 2017;376:417-28.

[6] Sooriakumaran P, Srivastava A, Shariat SF, et al. A multinational, multi-institutional study comparing positive surgical margin rates among 22393 open, laparoscopic, and robot-assisted radical prostatectomy patients. Eur Urol 2014;66:450-6.

[7] Mohler JL, Lee RJ, Antonarakis ES, et al., Prostate Cancer. Version 2.2019. National Comprehensive Cancer Network Clinical Practice Guidelines in Oncology. 2019.

[8] Mottet N, van den Bergh RCN, Briers E, et al., EAU - ESTRO - ESUR SIOG Guidelines on Prostate Cancer. Retrieved from: https://uroweb. org/guideline/prostate-cancer/ July 11, 2019.

[9] Thompson IM, Tangen CM, Paradelo J, et al. Adjuvant radiotherapy for pathological T3NOMO prostate cancer significantly reduces risk of metastases and improves survival: long-term followup of a randomized clinical trial. J Urol 2009;181:956-62.

[10] Bolla M, van Poppel H, Tombal B, et al. Postoperative radiotherapy after radical prostatectomy for high-risk prostate cancer: long-term 
results of a randomised controlled trial (EORTC trial 22911). Lancet 2012;380:2018-27.

[11] Wiegel T, Bartkowiak D, Bottke D, et al. Adjuvant radiotherapy versus wait-and-see after radical prostatectomy: 10-year followup of the ARO 96-02/AUO AP 09/95 trial. Eur Urol 2014;66:243-50.

[12] Fitzmaurice GM, Laird NM, Ware JH. Applied longitudinal analysis. Hoboken, NJ: John Wiley \& Sons; 2004.

[13] Hoffman KE, Nguyen PL, Chen MH, et al. Recommendations for postprostatectomy radiation therapy in the United States before and after the presentation of randomized trials. J Urol 2011;185:116-20.

[14] Hwang WL, Tendulkar RD, Niemierko A, et al. Comparison between adjuvant and early-salvage postprostatectomy radiotherapy for prostate cancer with adverse pathological features. JAMA Oncol 2018;4:e175230.

[15] Ost P, De Troyer B, Fonteyne V, Oosterlinck W, De Meerleer G. A matched control analysis of adjuvant and salvage high-dose postoperative intensity-modulated radiotherapy for prostate cancer. Int J Radiat Oncol Biol Phys 2011;80:1316-22.

[16] Mishra MV, Scher ED, Andrel J, et al. Adjuvant versus salvage radiation therapy for prostate cancer patients with adverse pathologic features: comparative analysis of long-term outcomes. Am J Clin Oncol 2015;38:55-60.

[17] Fossati N, Karnes RJ, Boorjian SA, et al. Long-term impact of adjuvant versus early salvage radiation therapy in pT3NO prostate cancer patients treated with radical prostatectomy: results from a multiinstitutional series. Eur Urol 2017;71:886-93.

[18] Pierorazio PM, Ross AE, Schaeffer EM, et al. A contemporary analysis of outcomes of adenocarcinoma of the prostate with seminal vesicle invasion (pT3b) after radical prostatectomy. J Urol 2011;185:1691-7.

[19] Lee HJ, Han JH, Lee DH, et al. Does bilateral seminal vesicle invasion at radical prostatectomy predict worse prognosis than unilateral invasion among patients with pT3b prostate cancers? Int J Urol 2016;23:758-63.

[20] Basch E, Dueck AC, Rogak LJ, et al. Feasibility assessment of patient reporting of symptomatic adverse events in multicenter cancer clinical trials. JAMA Oncol 2017;3:1043-50.

[21] Atherton PJ, Burger KN, Pederson LD, Kaggal S, Sloan JA. Patient reported outcomes questionnaire compliance in Cancer Cooperative Group Trials (Alliance N0992). Clin Trials 2016;13:612-20.

[22] Kishan AU, Shaikh T, Wang PC, et al. Clinical outcomes for patients with Gleason score 9-10 prostate adenocarcinoma treated with radiotherapy or radical prostatectomy: a multi-institutional comparative analysis. Eur Urol 2017;71:766-73.

[23] Peacock M, Quirt J, Morris WJ, et al. Population-based 10-year event-free survival after radical prostatectomy for patients with prostate cancer in British Columbia. Can Urol Assoc J 2015;9:409-13.

\title{
www.esui19.org
}

ESUI19

\section{8th Meeting of the EAU Section of Urological Imaging}

\author{
14 November 2019, Vienna, Austria
}

In conjunction with the 11th European Multidisciplinary Congress on Urological Cancers :o: EMUC19
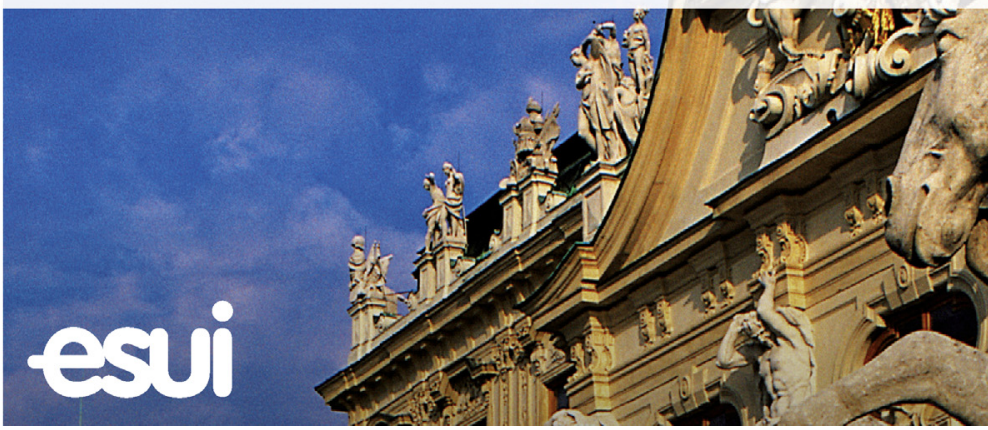

Less is more: What's really needed in imaging 